\title{
Analysis of Clinical and Radiological Outcomes of Posterior Dynamic Stabilization for Direct Repair of Bilateral L5 Pars Interarticularis Defect: Dynamic Assessment of Pars Fusion
}

\author{
Kenan $\mathrm{KIBICl}^{1}$, Berrin EROK ${ }^{2}$ \\ ${ }^{1}$ Altinbas University School of Medicine, Bahcelievler Medical Park Hospital, Department of Neurosurgery, Istanbul, Turkey \\ ${ }^{2}$ Cihanbeyli State Hospital, Department of Radiology, Konya, Turkey
}

Corresponding author: Berrin EROK drberrinerok@hotmail.com

\section{ABSTRACT}

AIM: To evaluate the satisfactory clinical and radiological outcomes of posterior dynamic stabilization for the direct repair of bilateral L5 pars interarticularis defects and pars fusion.

MATERIAL and METHODS: This is a retrospective evaluation of postoperative follow-up results of 13 patients with bilateral L5 pars interarticularis defects without spondylolisthesis. The patients underwent dynamic transpedicular stabilization between 2013 and 2018. Our surgical criteria included unilateral or bilateral L5 spondylolysis; excessive low back pain; low back pain accompanied by leg pain without neurological findings; symptoms lasting at least six months despite conservative treatments, age <50 years; and lack of significant adjacent disc degeneration.

RESULTS: There were seven female and six male patients with a mean age of 38.9 years. All patients achieved satisfactory postoperative results during a mean follow-up period of 22 months. Preoperative visual analog scale score and Oswestry Disability Index value were $8.85 \pm 0.69$ and $54.46 \pm 7.62$, respectively, which decreased to $1.31 \pm 0.48$ and $9.85 \pm 3.51$, respectively, at the postoperative $24^{\text {th }}$ month. In all patients, the bony fusion of the pars interarticularis at the stabilized segment was confirmed on the computed tomography scan at an average of 22 months postoperatively.

CONCLUSION: The posterior dynamic system ensures that the spine moves within physiological limits and carries the load by sharing it with the spine. The advantages of direct pars repair using our technique are the restoration of the posterior structures' normal anatomy, protection of the functional mobility segment, and early functional recovery without degeneration in the adjacent segment. Therefore, when there is no significant instability, patients with spondylolysis can be treated with posterior dynamic stabilization techniques with satisfactory clinical and radiological results.

KEYWORDS: Posterior dynamic stabilization, Pars interarticularis, L5 spondylolysis

\section{INTRODUCTION}

$\mathrm{U}$ nilateral or bilateral anatomical defects involving the pars interarticularis, the bridge of bone that connects bilateral superior and inferior articular processes at each vertebral level, are called spondylolysis. This condition is commonly known as pars interarticularis defect or, more simply, the pars defect. It can involve one or both sides of one or more vertebral bodies. These defects are observed almost exclusively in the lower lumbar spine, most frequently at the
L5 level (8). It is frequent among individuals engaged in athletic sports. In the general population, the incidence of these defects is $3 \%-11.3 \%$, but it is much higher among athletes (5). Patients may be asymptomatic; however, those with symptoms of spondylolysis generally present with progressive low back pain that is frequently aggravated by hyperextension and twisting of the spine with or without leg pain (35). Neurological signs are rare unless there is nerve root compression caused by accompanying spondylolisthesis. The diagnosis in patients with spondylolysis is made generally after a trauma or during 
examinations for chronic low back pain. Clinical examinations are nonspecific, so imaging modalities are the mainstay in the diagnosis. Lateral and oblique radiographs can be used initially to evaluate patients with low back pain and suspicion of spondylolysis. Lateral views are more sensitive for the detection of pars fractures; however, oblique views are more specific. The defect can be visualized as a "collar" around the neck of the Scottie dog, indicating a nondisplaced pars fracture on conventional oblique radiographs. However, due to the low sensitivity of bidirectional radiographs, it is difficult to make a diagnosis that can lead to incorrect treatment approaches (26). Computed tomography (CT) is more sensitive in detecting pars interarticularis defects, especially when they are unilateral and nondisplaced. Conservative approaches, such as physical therapy and corset application, are first-line treatments. Although most patients respond well to these approaches, some patients may require surgical treatment for persistent pain or neurological findings that do not respond to conservative treatments (32). In this study, we report our findings of direct pars repair with posterior dynamic stabilization in 13 patients with L5 spondylolysis without spondylolisthesis.

\section{MATERIAL and METHODS}

We included 13 patients (7 females and 6 males) with L5 pars interarticularis defects without spondylolisthesis who underwent posterior dynamic transpedicular stabilization between 2013 and 2018, and retrospectively evaluated their postoperative follow-up data (Tables I, II). Our surgical criteria were as follows:

1. Unilateral or bilateral L5 spondylolysis

2. Excessive low back pain or low back pain accompanied by leg pain without neurological findings

3. Symptoms lasting at least six months despite conservative treatments

4. Age $<50$ years

5. Lack of significant adjacent disc degeneration (L4-L5 disc)

Table I: UCLA Grading Scale for Intervetebral Disc Degeneration

\begin{tabular}{lccc}
\hline & Disc Space Narrowing & Osteophytes & Endplate Sclerosis \\
\hline Grade I & - & - & - \\
\hline Grade II & + & - & - \\
\hline Grade IIII & $+/-$ & + & - \\
\hline Grade IV & $+/-$ & $+/-$ & + \\
\hline
\end{tabular}

Grade is based upon the most severe radiographic finding evident on plain radiographs; + (present), - (absence), +/- (either present or absent).

Table II: Demographic Data of 13 Patients with Spondylolysis and Scores on the VAS in the Lower back and Lower Extremity Preoperatively and at Last Follow-up

\begin{tabular}{|c|c|c|c|c|c|c|c|c|c|c|}
\hline No & Age & Gender & Location & $\begin{array}{l}\text { UCLA } \\
\text { Grading } \\
\text { Scale }\end{array}$ & $\begin{array}{l}\text { Discogenic } \\
\text { Pain }\end{array}$ & $\begin{array}{l}\text { Segmental } \\
\text { Instability }\end{array}$ & $\begin{array}{l}\text { Preoperative } \\
\text { VAS } \\
\text { Score }\end{array}$ & $\begin{array}{l}\text { Last } \\
\text { Follow-up } \\
\text { VAS Score }\end{array}$ & $\begin{array}{c}\text { Preoperative } \\
\text { ODI } \\
\text { Score }\end{array}$ & $\begin{array}{l}\text { Last } \\
\text { Follow-up } \\
\text { ODI Score }\end{array}$ \\
\hline 1 & 25 & M & L5 & I & back & + & 9 & 1 & 58 & 8 \\
\hline 2 & 49 & $\mathrm{~F}$ & L5 & II & back / leg pain & + & 8 & 2 & 44 & 8 \\
\hline 3 & 40 & $\mathrm{~F}$ & L5 & II & back & + & 9 & 2 & 58 & 16 \\
\hline 4 & 44 & M & L5 & II & back / bilateral leg pain & + & 10 & 1 & 64 & 8 \\
\hline 5 & 49 & $\mathrm{~F}$ & L5 & II & back / bilateral leg pain & + & 8 & 1 & 44 & 8 \\
\hline 6 & 40 & $\mathrm{~F}$ & L5 & II & back / leg pain & + & 10 & 2 & 64 & 16 \\
\hline 7 & 39 & $\mathrm{~F}$ & L5 & II & back / bilateral leg pain & + & 9 & 1 & 58 & 8 \\
\hline 8 & 42 & M & L5 & II & back / bilateral leg pain & + & 9 & 2 & 58 & 16 \\
\hline 9 & 32 & M & L5 & II & back / leg pain & + & 8 & 1 & 44 & 8 \\
\hline 10 & 29 & M & L5 & 1 & back / leg pain & + & 9 & 1 & 56 & 8 \\
\hline 11 & 33 & $\mathrm{~F}$ & L5 & ॥ & back / leg pain & + & 9 & 1 & 58 & 8 \\
\hline 12 & 41 & $\mathrm{~F}$ & L5 & II & back / bilateral leg pain & + & 9 & 1 & 58 & 8 \\
\hline 13 & 43 & M & L5 & ॥ & back / leg pain & + & 8 & 1 & 44 & 8 \\
\hline
\end{tabular}

ODI: Oswestry disability index, VAS: Visual analogue scale. 
We excluded patients with deep osteoporosis, vertebral fracture, scoliosis, ankylosing spondylitis, advanced spinal instability, and obesity. Anteroposterior and lateral (AP/L) radiographs were evaluated first, and subsequently, CT scans were used for confirmation and more detailed examination. In all our study patients, spondylolysis was at the L5 level. Disc degeneration was evaluated by magnetic resonance (MR) imaging and graded using the UCLA Grading Scale for intervertebral disc degeneration. Two patients had grade 1 degeneration, and 11 patients had grade 2 degeneration in the surgical segment disc (L5-S1 disc) (Table I). There was no significant degeneration in the adjacent segment disc (L4-L5 disc).

\section{Surgical Method}

All patients underwent surgery under general anesthesia. A single dose of prophylactic antibiotics (IV $1 \mathrm{~g}$ cefazoline) was administered $30 \mathrm{~min}$ before making the skin incision. The patient was placed in the prone position on the operation table by supporting the thorax and pelvis using gel pillows. Level detection and markings were performed using the C-armed scope device. After passing the skin, subcutaneous tissue, and thoracolumbar fascia, the paravertebral muscles were peeled off, exposing the bilateral L4-5 and L5-S1 facet joints. Pedicle entries were marked again, using the scope device. Polyaxial titanium screws (Figure 1) suitable for the pedicle length and diameter, which were measured from preoperative radiographs, were inserted into the vertebra corpus. After applying the same procedure for the opposite side screws, the patient was brought to the neutral position. The system was locked with screw caps by introducing the dynamic rod into the screw heads. In all our surgeries, we used the flexmotion spine rod (Tasarimmed, Istanbul) made from polyether ether ketone (PEEK) optima polymer (Invibio Biomaterial Solutions, UK) (PEEK-OPTIMA ${ }^{\mathrm{TM}}$ ) (Figure 2). None of the patients underwent bone fusion. Hemostasis was achieved using bipolar cautery, and a hemovac drain was placed in the surgical area. The fascia, subcutaneous tissue, and skin were closed using resorbable vicryl. The drain was withdrawn the next day.

The entire procedure was completed in 2-3 hours. Patients were mobilized the day after surgery without bracing. They remained in the hospital for an average of three days postoperatively. After discharge, they were advised to rest at home for one week, followed by walking on flat ground for 30 minutes to 1 hour till the end of 3 weeks. At the end of 3 weeks, by making their first controls, they could return to normal activities with limited waist exercises.

\section{Follow-up of Patients}

Postoperative radiological assessments included lumbosacral $\mathrm{AP} / \mathrm{L}$ radiographs, lumbosacral computed tomography $(\mathrm{CT})$ scans, and magnetic resonance imaging (MRI). All patients were followed for at least 18 months at outpatient visits (18-26 months). Preoperative and postoperative low back pain and leg pain were evaluated using the visual analog scale (VAS) $(0=$ no pain, 10 = the most severe pain). The value indicated by the patient was interpreted as his/her pain level (37). The Oswestry

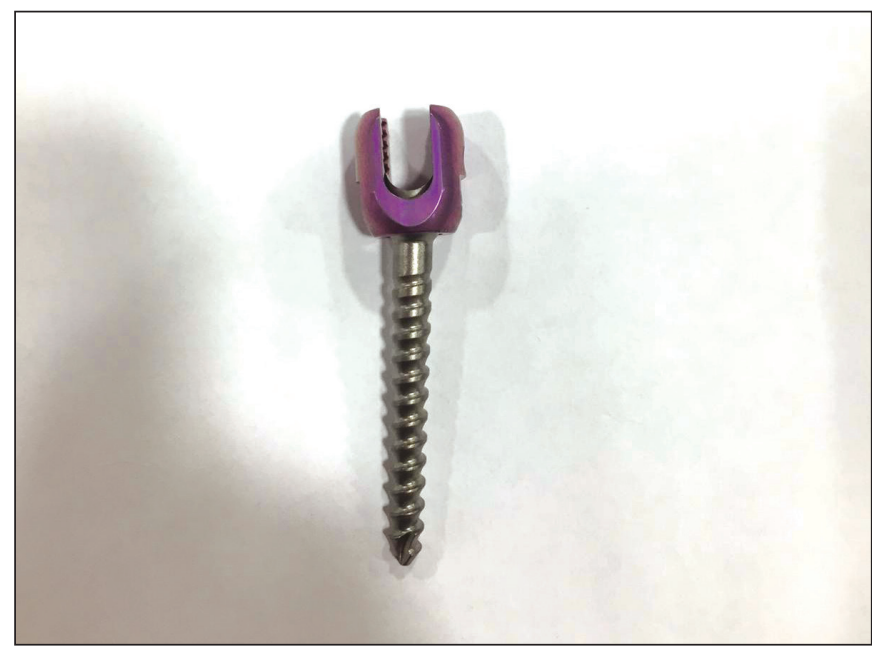

Figure 1: The polyaxial screw.

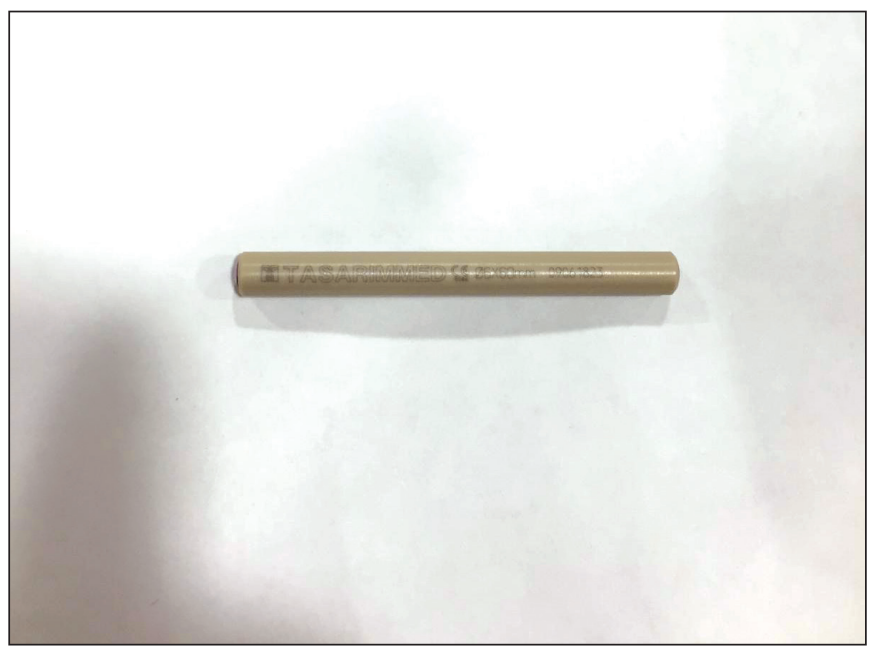

Figure 2: The dynamic PEEK rod.

Disability Index (ODI) was used to determine the extent to which the low back pain affected their daily life activities (9). The preoperative values and the final follow-up values of VAS and ODI scores were considered for statistical analysis. The first control was done by lumbosacral AP/L radiography at postoperative $3^{\text {rd }}$ week, and then radiological imaging was performed using serial CT scans starting from the $3^{\text {rd }}$ month. The bony fusion in the postoperative pars was evaluated. The dynamic transpedicular system was removed at postoperative $18^{\text {th }}$ month in a male patient and the $48^{\text {th }}$ month in a female patient at their request due to itching in the entire body in the male patient and excessive sweating in one half of the body accompanied by emotional disturbances in the female patient. Dermatology consultation was requested for the male patient before removing the implants. A prick test consisting of 10 parameters indicated negative results. As the patient's complaints did not regress despite medical treatment, after confirming sufficient fusion by radiographic examinations, the implants were removed since his symptoms may be due to titanium hypersensitivity. The complaints disappeared entirely during the postoperative $4^{\text {th }}$ week. In the female patient, the 
implants were removed, but the sweating issue persisted.

\section{Statistical Analysis}

All data are presented as mean and standard deviation. A paired $t$-test was used to evaluate the preoperative and final follow-up VAS and ODI scores to analyze the pain and functional capacity, respectively. Statistical significance was defined at $p<0.05$.

\section{- RESULTS}

Our study subjects consisted of seven female and six male patients with a mean age of 38.9 years. Postoperatively, all patients achieved satisfactory results without any complication. The outcomes were evaluated at 3, 6, 12, 18, and 24 months, with a mean follow-up duration of 22 months (range, 18-26 months). Significant pain relief was expressed by all patients and confirmed by the VAS and ODI scores. The preoperative VAS and ODI values were $8.85 \pm 0.69$ and 54.46 \pm 7.62 , respectively, which decreased to $1.31 \pm 0.48$ and 9.85 \pm 3.51 , respectively, at the postoperative $24^{\text {th }}$ month (Table III). In all our patients, the bony fusion of the pars interarticularis in the stabilized segment was confirmed on CT scans at an average of 22 months postoperatively. Moreover, the followup MR imaging findings revealed neither degeneration in the adjacent disc nor further degeneration in the surgical segment disc. Also, in young patients, we noted some rehydration in the surgical segment disc.

\section{Case Illustration}

A 24-year-old male patient visited our hospital with a complaint of low back pain for approximately 1.5 months that progressively worsened. He was a student who was engaged in bodybuilding. He had received all conservative treatments, including drugs, corset application, and physical treatments, but there was no benefit. Physical examination disclosed painful lumbar movements, negative Lasegue signs, and normal neurological findings. Lumbar CT scans revealed bilateral pars interarticularis defect at the L5 vertebra (Figure $3 \mathrm{~A}, \mathrm{~B})$. Lumbar MR imaging performed at another institution showed a mild degeneration and bulging in the L5-S1 disc (Figure 3C, D). After confirming spondylolysis was the source of the pain based on the use of a local anesthetic agent, the patient underwent surgery. Bilateral transpedicular screws were placed in the L5 and S1 vertebrae, and the system was connected using a PEEK dynamic rod. He was discharged home three days after the surgery and rested at home for one week. At the end of three weeks, by making the first controls by lumbosacral $A P / L$ radiography (Figure $4 A, B$ ), he could resume normal activities with limited waist exercises. We then examined him at $3,6,12$, and 18 months for evaluating pain recovery and pars fusion as well as the surgical and adjacent disc status. The patient had no complaints during these follow-up examinations. In the follow-up radiological imaging, pars fusion was detected on serial CT scans (Figure 5A-H). Lumbosacral MR imaging, performed at postoperative $12^{\text {th }}$ month, revealed some rehydration in the surgical segment disc

Table III: VAS and ODI Scores of the Patients

\begin{tabular}{lcccc} 
& & & \multicolumn{2}{c}{ Postoperative } \\
\cline { 3 - 5 } & Preoperative & 6-Month & 12-Month & 24-Month \\
\hline VAS & $8.85 \pm 0.69$ & $2.00 \pm 0.71$ & $1.31 \pm 0.48$ & $1.31 \pm 0.48$ \\
\hline ODI & $54.46 \pm 7.62$ & $25.85 \pm 1.72$ & $20.62 \pm 0.96$ & $9.85 \pm 3.51$ \\
\hline
\end{tabular}

VAS: Visual analogue scale, ODI: Oswestry disability index.
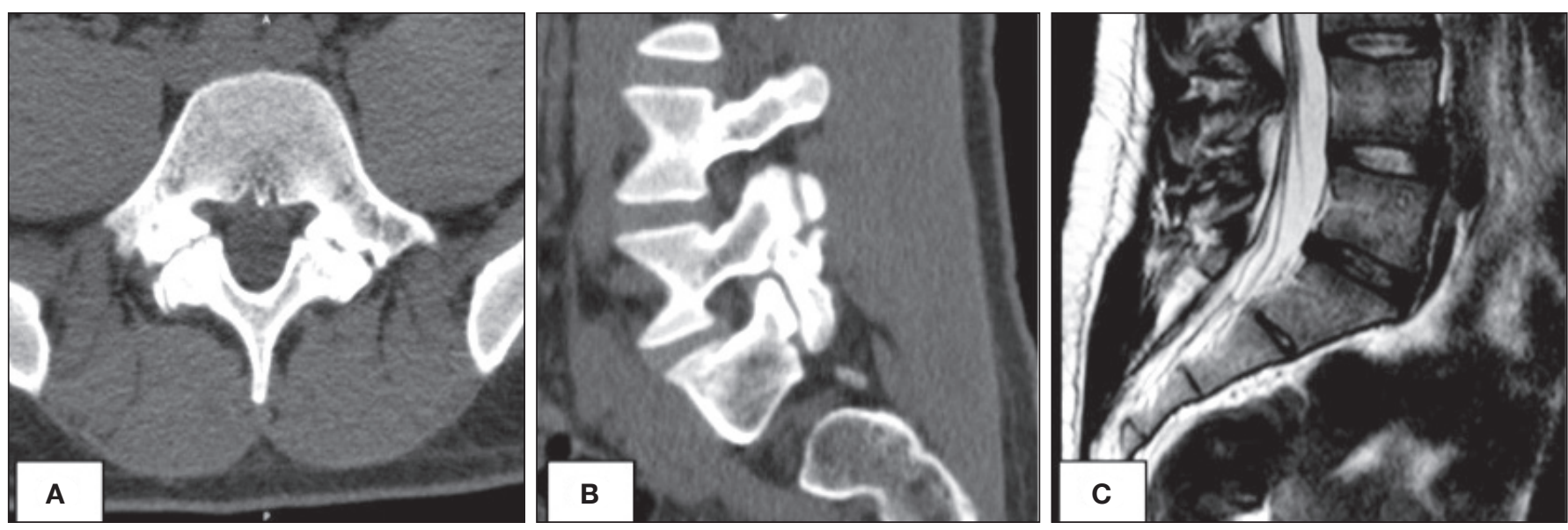

Figure 3: A 24-year-old man with chronic low back pain. Preoperative CT scan; axial (A) and sagittal (B) images demonstrating bilateral pars interarticularis defect of L5 vertebra. Preoperative sagittal T2W MR image (C) shows mild degeneration and bulging of the surgical segment disc (L5-S1). 
and no degeneration in the adjacent segment disc compared to the findings in the preoperative MR imaging (Figure 6A, B). The system was removed at the $18^{\text {th }}$ month when the patient complained of itching in the entire body, which resolved entirely (Figure 7A, B).

\section{DISCUSSION}

In spondylolysis, the pars may be congenitally defective or it is damaged by overuse. The exact cause remains unknown, but the most commonly accepted theory is repetitive stress due to axial loading, hyperextension, and rotation, which weakens the pars interarticularis and causes fatigue fractures in genetically susceptible individuals. Flattening of lumbar lor- dosis or hyperlordosis may cause abnormal stresses on the spine that may cause these anatomical defects, including a crack, fracture, or separation $(15,35)$. High pelvic incidence (PI) and sacral slope (SS) $\left(>40^{\circ}\right)$ values increase shear forces on the L5-S1 disc and increase strain on the pars interarticularis of the L5 vertebra during the development of spondylolysis and low-grade developmental spondylolisthesis. In contrast, in cases with low PI and SS values, lytic defects are reportedly caused by recurrent impingements on the L5 vertebra from extension movements of the L4-S1 vertebra (28). Hyperextension forces are often responsible for the condition. Imbalances in spinal muscle strength increase the suppressor or rotational forces biomechanically and can exacerbate the clinical situation. These imbalances bring the pelvis to the an-
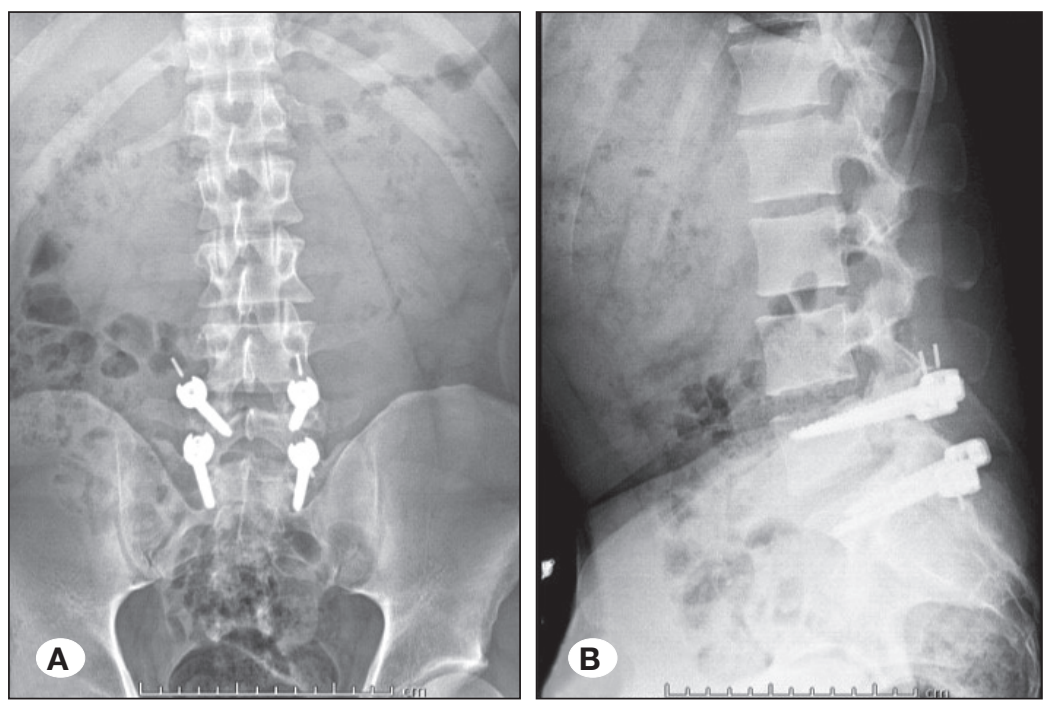

Figure 4: Posterior dynamic stabilization with PEEK rods, postoperative $3^{\text {rd }}$-month lumbosacral radiograms; anteroposterior (AP) (A) and lateral (B) images showing transpedicular screw and radiolucent dynamic PEEK rods.
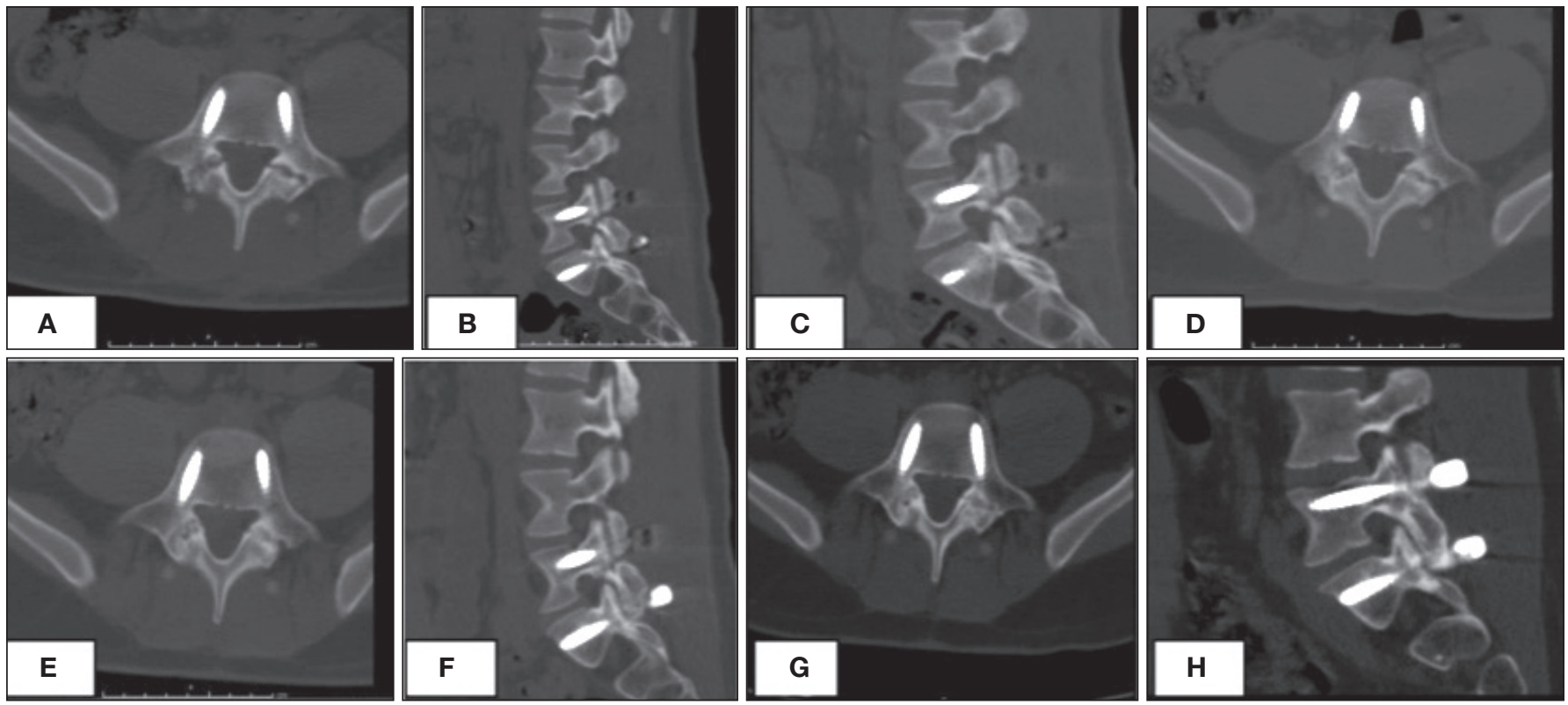

Figure 5: Posterior dynamic stabilization with PEEK rods, lumbosacral computerized tomography scans, postoperative $3^{\text {rd }}$ month; axial (A) and sagittal (B), postoperative $6^{\text {th }}$ month; axial (C) and sagittal (D), postoperative $12^{\text {th }}$ month; axial (E) and sagittal (F), postoperative $18^{\text {th }}$ month; axial $(\mathbf{G})$ and sagittal $(\mathbf{H})$ images; demonstrating a progressive fusion of the bilateral pars interarticularis defects of L5 vertebrae. 

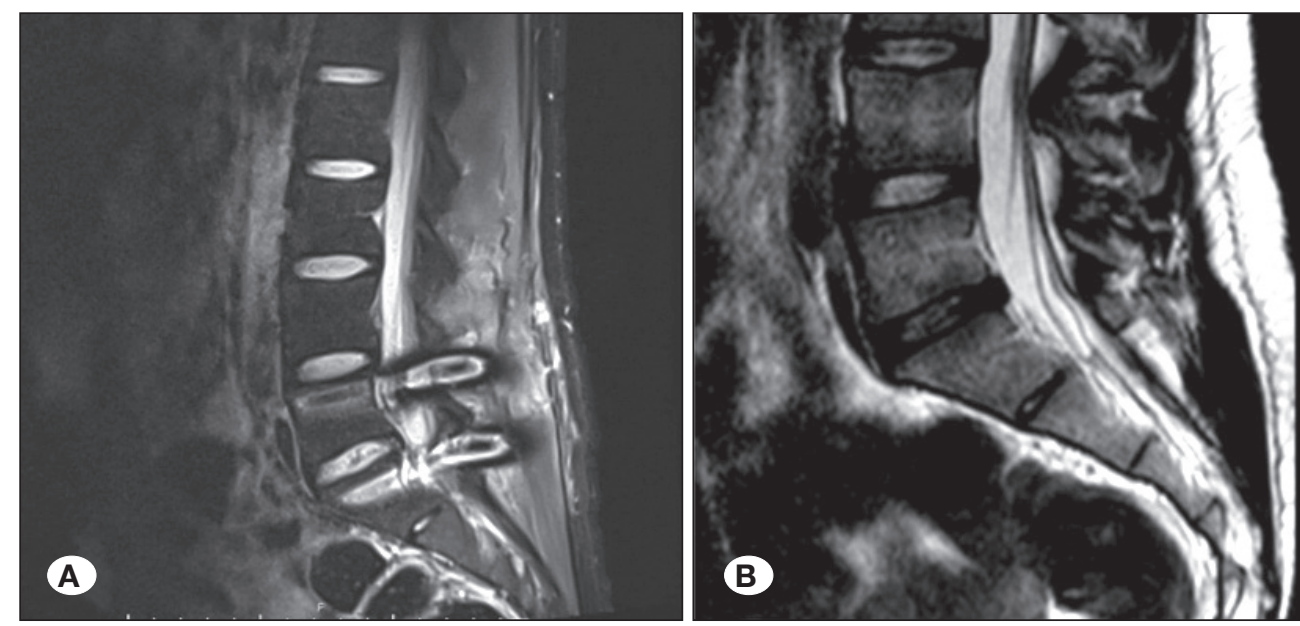

Figure 6: Posterior dynamic stabilization with PEEK rods; postoperative $12^{\text {th }}$ month, sagittal T2W lumbosacral MR image shows (A) some rehydration in the surgical segment disc (L5-S1) and no further degeneration in the adjacent segment disc (L4-L5) compared with the preoperative sagittal T2W lumbosacral MR image shows (B).
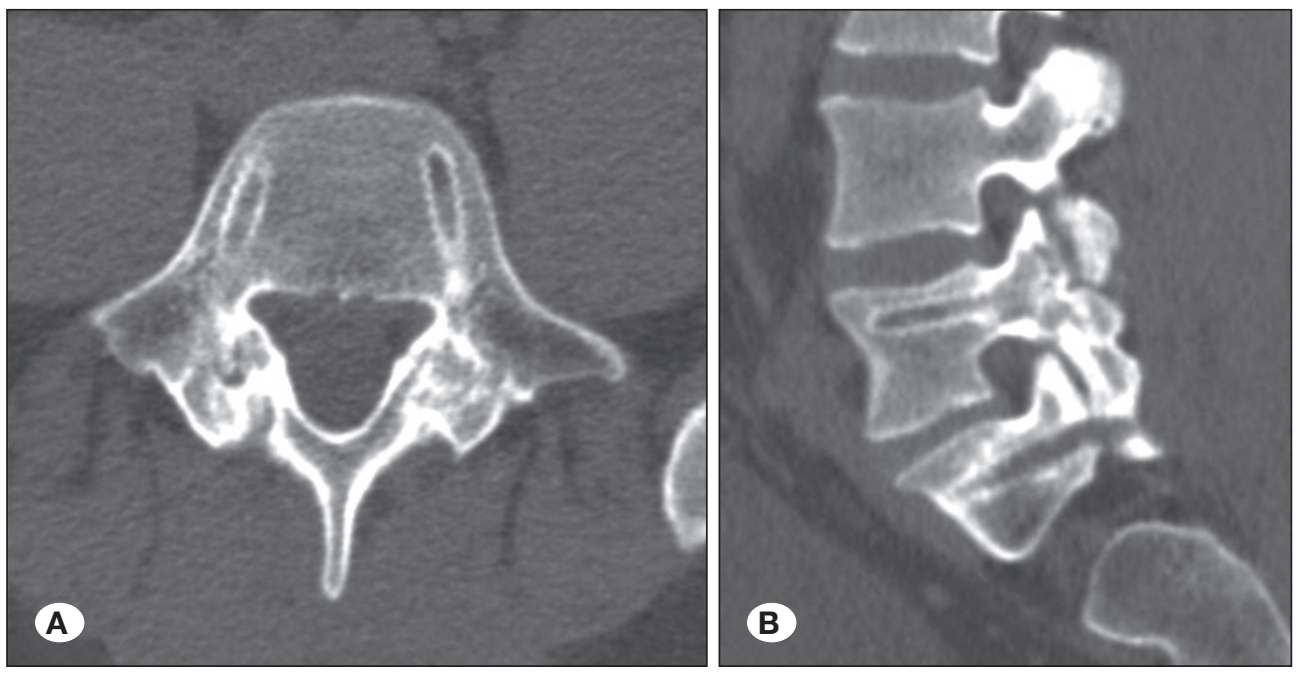

Figure 7: Lumbosacral CT scan performed three months after the removal of the dynamic lumbar system, axial (A), and sagittal (B) images demonstrating bilateral pars interarticularis fusion.

terior pelvic tilt position, which forces the lumbar region to hyperextension. Weak waist extensors and stretched hamstrings, hip flexors, and lumbar rotational muscles promote spondylolysis development. Spondylolysis is common in individuals who are engaged in sports involving athletic activity, especially diving. Many spondylolysis patients report a previous or current history of athletic activity (31). In our study, three patients were involved in such sports, including weightlifting, long jump, and ultra trailing. Furthermore, genetic differences that may be associated with weak joining points in the bony structure and a relatively low bone density make this area susceptible to damage. Spondylolysis occurs most frequently in the $L 5(85 \%-95 \%)$ and $L 4(5 \%-15 \%)$ vertebrae. It is much less common at higher spinal levels. Some studies indicate that, in the lower lumbar vertebra, the ossification center, originating in the pars region, results in uneven distribution of trabeculation and cortication. This uneven distribution may increase susceptibility to stress fractures. However, in the upper lumbar levels, the ossification center originating at the end of the pedicle results in a more uniform trabeculation throughout the pars interarticularis (29). Spondylolysis may be unilateral or bilateral, and the unilateral cases generally occur on the left side (34). However, most cases are bilateral, possibly because of increased stress on the contralateral pedicle, in patients with spondylolysis (30). The involvement of multiple levels may occur, but it is rare. All our study patients were aged $<50$ years and had bilateral L5 spondylolysis without spondylolisthesis. Both CT scans and MR imaging are more sensitive than radiographs for diagnosis. CT is the gold standard modality, especially for detecting unilateral and nondisplaced defects $(2,12)$. For evaluating more progressive disease, CT is excellent; however, MR imaging is better for diagnosing early-stage disease in which cortical disruption has not yet occurred (35). Various studies have reported on the surgical treatment of spondylolysis, but several issues remain debatable. One of the most important discussions is about cases requiring surgical management. The indications for surgical treatment could include permanent low back and/or leg pain, waist stiffness, and hamstring irritability. Initially, the bone grafting method was suggested for the surgical treatment of spondylolysis (16). Then, the single screw treatment for pars fracture was defined, which was found to be successful in $88 \%$ of the patients (6). In another study, the wiring technique was successful in $80 \%$ of 22 cases (4). The modified hook and 
transpedicular screw technique was also described as a treatment approach and has became widespread following advances spinal implant developments, with excellent clinical and radiological results (21). Another report indicated that the possibility of direct repair should be considered in symptomatic patients with pars interarticularis pseudoarthrosis (10). A direct repair can be performed in both bilateral and unilateral lesions. Although segmental fusion has been used to treat lumbar degenerative diseases for years, whether a lumbosacral fusion should be performed or not has been debated in terms of clinical outcomes. If fusion is achieved, there is a significant improvement. However, several studies have demonstrated that a mismatch exists between the radiological fusion and clinical success. This seems to be because of the increased risk of pseudoarthrosis and adjacent segment degeneration in fusion surgeries (22). Disc degeneration is the most common among these degenerative changes adjacent to the fused segment. Several in vivo and in vitro studies indicated that the degenerative processes in the adjacent segment are accelerated due to the changes in the biomechanics of the spine from hypermobility, facet loading, and increased intradiscal pressure after fusion (17-19). Moreover, disc degeneration at the level of the pars interarticularis defect (surgical segment disc) is generally an accompanying disorder in patients with spondylolysis. Loss of bone integrity in the pars interarticularis can increase the load on the disc at the level of spondylolysis and may cause degeneration $(15,35)$. Some recently published studies have shown that dynamic stabilization, using a dynamic rod system, provides stability similar to rigid systems but without the complication of spinal fusion (12). Clinicians have begun applying this technique to improve segmental stability and reduce the morbidity associated with arthrodesis by maintaining physiological segmental movement (13). Using dynamic systems ensures that the spine moves within physiological limits, with segmental load distribution preventing the disc from being more degenerated; it also ensures that the disc is rehydrated. Several studies have demonstrated improvements in disc hydration at the treated level on follow-up MR imaging scans $(3,23)$. The degenerated disc began returning to normal when the disc did not degenerate excessively and maintained its repair capacity in patients treated with dynamic systems (25). In our study patients, no further degeneration occurred in the surgical segment disc. Moreover, in young patients, there was some disc hydration, which is consistent with the abovementioned findings reported in the literature. Therefore, it is better to restore the normal anatomy having mobile segments in physiological limits, especially in young patients. MR imaging is used in the postoperative follow-up of disc changes. However, metal implants can cause susceptibility artifacts in relation to the type of metal and its position relative to the primary magnetic field and MR sequences (33). Spin-echo sequences and some parameter changes, such as slew rate, help decrease these artifacts (20). The following advantages of posterior dynamic stabilization can be listed: maintaining the controlled movement of the unstable segment, slowing down of the adjacent disc degeneration by reducing the additional load exerted on the adjacent segment, reducing facet joint degeneration and hypertrophy by reducing the burden on the degenerated facet joints, and preventing the problems such as instrument failure and pseudoarthrosis that may occur after fusion surgeries. Furthermore, semi-rigid rod systems, including PEEK and carbon fiber rods, can be used in dynamic stabilization. These systems provide less rigidity in the spine than metal systems, thus reducing the rate of screw loosening and increasing the fusion rates of the pars interarticularis (24). We performed stabilization in all our patients using flex-motion rods from PEEK-OPTIMA ${ }^{\mathrm{TM}}$ for supporting the transpedicular system. PEEK-OPTIMA ${ }^{\mathrm{TM}}$ rods are durable and highly resistant to cracks and fatigue. These rods have a bone-like modulus that helps minimize stress shielding and stimulates bone healing. Flex-motion spine rods have superior properties than metallic dynamic pedicle screws. Although the dynamic pedicle screws can produce motion in only one plane, the flex-motion spine rod is in the range of motion in all planes (11). Unlike metallic implants, the PEEK-OPTIMA ${ }^{\mathrm{TM}}$ spinal rods provide artifact-free imaging when using X-ray, CT, and MR imaging techniques (1). In the postoperative follow-up, both radiographs and CT scans can evaluate the position and unity of the instrumentation. However, CT is better for these evaluations and the detection of complications, including, for example, pedicle fractures (1). In our study patients, lumbosacral CT was used in the postoperative follow-up evaluations. This surgery's primary aim is to protect spinal stability by maintaining physiological motion and eliminating the complications associated with unnatural movements created by spinal fusion. However, the pars interarticularis of the stabilized segment undergoes fusion in most cases. In all our patients, the pars interarticularis was fused in an average of 22 months. Moreover, in our clinical practice, the pars interarticularis of the stabilized segment undergoes fusion more commonly and rapidly in dynamic stabilization than in rigid systems. This result can be explained by the absence of drastic changes in the physiological stress forces that occur in the rigid systems as a result of the created definitive curvature (27). In dynamic stabilization, the patient's particular spinal balance is maintained without abrupt elimination of the physiological stress forces. Patients' complaints, neurological examination findings, radiological evaluation of the fusion area, and surgical instruments are important in deciding whether the screw-rod system can be removed. If the complaints of the patient disappear and the radiological imaging reveals fusion, the system can be removed. In our study patients, two dynamic systems were removed upon the patient's request, one in the $18^{\text {th }}$ month and another in the $48^{\text {th }}$ month. Despite the low risk of allergies, it is necessary to consider the hypersensitivity associated with the presence of titanium in patients with itchy lesions, as in our case (14). In our other case, the system was removed upon the patient's request. In case the patient's complaints disappear, and radiological imaging reveals fusion, the system can be removed. In both of our cases, pars fusion was confirmed on CT scans. The dynamic stabilization turned out to be a method that should definitely be preferred over fusion surgeries, with the consideration of the time to return to daily life, the length of hospital stay, the postoperative pain, and the hospital expenses. It is the primary solution in the surgical treatment of spondylolysis without spondylolisthesis. It is not an intermediate solution before fusion surgeries. The 
results of clinical applications of existing systems should also guide the biomechanical studies. Although studies show that the combined use of dynamic screws and dynamic rods provides more effective stabilization in the spine, our study included only patients with spondylolysis without spondylolisthesis (7). We anticipate that our clinical results would set an example for these studies.

\section{CONCLUSION}

Different dynamic systems are used worldwide, but studies evaluating their safe uses are limited. The uses of these dynamic systems vary on a regional basis with different opinions. The dynamic lumbar system applied in our patients is a rod dynamic stabilization system that allows movement in all the planes. The advantages of direct pars repair with our technique are the restoration of the normal anatomy of posterior structures, protection of the functional mobility segment, and early functional recovery without any degeneration in the adjacent segment disc. Radiological imaging plays a vital role in the postoperative evaluation of the position and unity of the instrumentation, the investigation of the suspected complications, and the assessment of the surgical and adjacent disc status along with the developing fusion of pars interarticularis. However, careful patient selection is essential for the success of this procedure. We believe this procedure should be limited to patients with spondylolysis without significant spondylolisthesis to achieve satisfactory clinical outcomes.

\section{REFERENCES}

1. Benezech J, Garlenq B, Larroque G: Flexible stabilisation of the degenerative lumbar spine using PEEK rods. Adv Orthop 2016:7369409, 2016

2. Berquist $\mathrm{TH}$ : Imaging of the postoperative spine. Radiol Clin $\mathrm{N}$ Am 44:407-418, 2006

3. Bordes-Monmeneu M, Bordes-Garcia V, Rodrigo-Baeza F, Saéz D: System of dynamic neutralization in the lumbar spine: Experience on 94 cases. Neurocirugia (Astur) 16:499-506, 2005

4. Bradford DS, Iza J: Repair of the defect in spondylolysis or minimal degrees of spondylolisthesis by segmental wire fixation and bone grafting. Spine 10:673-679, 1985

5. Brooks BK, Southam SL, Mlady GW, Logan J, Rosett M: Lumbar spine spondylolysis in the adult population: Using computed tomography to evaluate the possibility of adult onset lumbar spondylosis as a cause of back pain. Skeletal Radiol 39(7):669-673, 2010

6. Buck JE: Direct repair of the defect in spondylolisthesis. Preliminary report. J Bone Joint Surg Br 52(3):432-437,1970

7. Erbulut D: "Kinematical and load sharing effect of a novel posterior dynamic stabilization system implanted in lumbar spine" in Proceedings of the $36^{\text {th }}$ Annual Meeting of the American Society of Biomechanics, Gaines ville, Fla, USA, August 2012.

8. Fredrickson BE, Baker D, McHolick WJ, Yuan HA, Lubicky JP: The natural history of spondylolysis and spondylolisthesis. J Bone Joint Surg Am 66(5):699-707, 1984
9. Fritz JM, Irrgang JJ: A comparison of a modified oswestry low back pain disability questionnaire and the quebec back pain disability scale. Phys Ther 81:776-788, 2001

10. Geissele AE, Ryan JB, Van Dam BE: Treatment of traumatic spondylolysis by amodified Scott technique. AAOS, $57^{\text {th }}$ Annual Meeting, 1990

11. Invibio Spine. PEEK-OPTIMA® Spinal Rods: A retrospective clinical study.

12. Iwamoto J, Abe H, Tsukimura Y, Wakano K: Relationship between radiographic abnormalities of lumbar spine and incidence of low back pain in high school rugby players: A prospective study. Scandinavian Journal of Medicine \& Science in Sports 15(3):163-168, 2005

13. Kaner T, Dalbayrak S, Oktenoglu T, Sasani M, Aydin AL, Ozer AF: Comparison of posterior dynamic and posterior rigid transpedicular stabilization with fusion to treat degenerative spondylolisthesis. Orthopedics 33(5), 2010

14. Kaya S, Karakose S, Onder H: A rare case: Titanium hypersensitivity. Van Tıp Derg 24(4):359-360, 2017

15. Khan SA, Sattar A, Khanzada U, Adel H, Adil SO, Hussain M: Facture of the pars interarticularis with or without spondylolisthesis in an adult population in a developing country: Evaluation by multidetector computed tomography. Asian Spine Journal 11(3):437-443, 2017

16. Kimura M: My method of filing the lesion with spongy bone in spondylolysis and spondylolistesis. Seikei Geka 19:285-296, 1968

17. Kumar A, Beastall J, Hughes J, Karadimas EJ, Nicol M, Smith F, Wardlaw D: Disc changes in the bridged and adjacent segments after Dynesys dynamic stabilization system after two years. Spine 33(26):2909-2914, 2008

18. Lee CK, Langrana NA: Lumbosacral spinal fusion. A biomechanical study. Spine 9(6):574-581, 1984

19. Lipson SJ: Degenerative spinal stenosis following old lumbosacral fusion. Orthop Trans 7:143, 1983

20. Marshmann LAG, Strong G, Trewhella M, Kasis A, Friesem T: Minimizing fer romagnetic artefact with metallic lumbar total disc arthroplasty devices at adjacent segments technical note. Spine 35(2):252-256, 2010

21. Morscher E, Gerber B, Fasel J: Surgical treatment of spondylolisthesis by bone grafting and direct stabilization of spondylolysis by means of a hook screw. Arch Orthop Trauma Surg 103:175-178, 1984

22. Murtagh RD, Quencer RM, Cohen DS, Yue JJ, Sklar EL: Normal and abnormal imaging findings in lumbar total disc replacement: Devices and complications. Radiographics 29:105-118, 2009

23. Oktenoglu T, Ozer AF, Sasani M, Kaner T, Canbulat N, Ercelen O, Sarioglu AC: Posterior dynamic stabilization in the treatment of lumbar degenerative disc disease: Two-year followup. Minim Invasive Neurosurg 53:112-116, 2010

24. Ormond DR, Albert L Jr, Das K: Polyetheretherketone (PEEK) rods in lumbar spine degenerative disease: A case series. Clin Spine Surg 29(7):E371-375, 2016 
25. Ozer AF, Oktenoglu T, Egemen E, Sasani M, Yilmaz A, Erbulut DU, Yaman O, MD, Suzer T: Lumbar single-level dynamic stabilization with semi-rigid and full dynamic systems: A retrospective clinical and radiological analysis of 71 patients. Clinics in Orthopedic Surgery 9:310-316, 2017

26. Pai VS, Hodgson B: Assessment of bony union following surgical stabilisation for lumbar spondylolysis: A comparative study between radiography and computed tomography. J Orthop Surg (Hong Kong) 14:17-20, 2006

27. Ricart O, Serwier JM: Dynamic stabilisation and compression without fusion using Dynesys for the treatment of degenerative lumbar spondylolisthesis: A prospective series of 25 cases. Rev Chir Orthop Reparatrice Appar Mot 94(7):619-627, 2008

28. Roussouly P, Gollogly S, Berthonnaud E, Labelle $H$, Weidenbaum M: Sagittalalignment of the spine and pelvis in the presence of L5-S1 isthmic lysis and low -grade spondylolisthesis. Spine 31(21):2484-2490, 2006

29. Sagi HC, Jarvis JG, Uhthoff HK: Histomorphic analysis of the development of the pars interarticularis and its association with isthmic spondylolysis. Spine 23(15):1635-1639; discussion 1640, 1998

30. Sairyo K, Katoh S, Sasa T, Yasui N, Goel VK, Vadapalli S, Masuda A, Biyani A, Ebraheim N: Athletes with unilateral spondylolysis are at risk of stress fracture at the contralateral pedicle and pars interarticularis: A clinical and biomechanical study. Am J Sports Med 33(4):583-590, 2005
31. Sakai T, Goda Y, Tezuka F, Takata Y, Higashino K, Sato M, Mase Y, Nagamachi A, Sairyo K: Characteristics of lumbar spondylolysis in elementary school age children. Eur Spine J 25(2):602-606, 2016

32. Snyder LA, Shufflebarger H, O'Brien MF, Thind H, Theodore N, Kakarla UK: Spondylolysis outcomes in adolescents after direct screw repair of the pars interarticularis. J Neurosurg Spine 21:329-333, 2014

33. Sofka CM: Optimizing techniques for musculoskeletal imaging of the postoperative patient. Radiol Clin N Am 44:323-329, 2006

34. Standaert CJ, Herring SA: Spondylolysis: A critical review (Review). Br J Sports Med 34:415-422, 2000

35. Syrmou E, Tsitsopoulos PP, Marinopoulos D, Tsonidis C, Anagnostopoulos I, Tsitsopoulos PD: Spondylolysis: A review and reappraisal. Hippokratia 14(1):17-21, 2010

36. Tawfik S, Phan K, Mobbs RJ, Rao PJ: The incidence of pars interarticularis defects in athletes. Global Spine J 10(1):89101, 2020

37. Williams A, Hoggart B: A review of three commonly used pain rating scales. J Clin Nurs Pain 14:798-804, 2005 